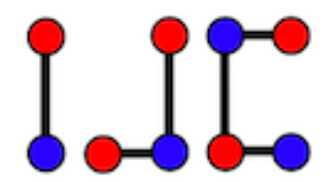

\title{
Local antimagic vertex coloring of unicyclic graphs
}

\author{
Nuris Hisan Nazula ${ }^{\mathrm{a}}$, Slamin ${ }^{\mathrm{b}}$, Dafik $^{\mathrm{a}}$ \\ ${ }^{a}$ Mathematics Education Study Program, University of Jember, Indonesia \\ ${ }^{b}$ Informatics Study Program, University of Jember, Indonesia \\ nuris.h.nazula@gmail.com, slamin@unej.ac.id,d.dafik@unej.ac.id
}

\begin{abstract}
The local antimagic labeling on a graph $G$ with $|V|$ vertices and $|E|$ edges is defined to be an assignment $f: E \rightarrow\{1,2, \cdots,|E|\}$ so that the weights of any two adjacent vertices $u$ and $v$ are distinct, that is, $w(u) \neq w(v)$ where $w(u)=\Sigma_{e \in E(u)} f(e)$ and $E(u)$ is the set of edges incident to $u$. Therefore, any local antimagic labeling induces a proper vertex coloring of $G$ where the vertex $u$ is assigned the color $w(u)$. The local antimagic chromatic number, denoted by $\chi_{l a}(G)$, is the minimum number of colors taken over all colorings induced by local antimagic labelings of $G$. In this paper, we present the local antimagic chromatic number of unicyclic graphs that is the graphs containing exactly one cycle such as kite and cycle with two neighbour pendants.
\end{abstract}

Keywords: local antimagic labeling, vertex coloring, unicyclic graphs, kite, cycle with two neighbour pendants Mathematics Subject Classification : 05C78, 05C15

DOI: $10.19184 /$ ijc.2018.2.1.4

\section{Introduction}

Let $G=(V, E)$ be a finite, simple, connected and undirected graph. The local antimagic labeling on a graph $G$ with $|V|$ vertices and $|E|$ edges is defined to an assignment $f: E \rightarrow$ $\{1,2, \cdots, m\}$ so that the weights any two adjacent vertices are distinct, that is, $w(u) \neq w(v)$ where $w(u)=\Sigma_{e \in E(u)} f(e)$ and $E(u)$ is the set of edges incident to $u$. Therefore, any local antimagic labeling induces a proper vertex coloring of $G$ where the vertex $u$ is assigned the color $w(u)$. The local antimagic chromatic number, denoted by $\chi_{l a}(G)$, is the minimum number of colors taken

Received: 06 Feb 2018, Revised: 09 May 2018, Accepted: 09 Jun 2018. 
over all colorings induced by local antimagic labelings of $G$. This concept was recently introduced by Arumugam et al. [1].

In the paper [1], Arumugam et al. presented the exact value of the local antimagic chromatic number of some families of graphs as follows.

- Complete graph $K_{n}$ on $n \geq 3$ vertices, $\chi_{l a}\left(K_{n}\right)=n$.

- $\operatorname{Star} K_{1, n-1}$ on $n \geq 3$ vertices, $\chi_{l a}\left(K_{1, n-1}\right)=n$.

- Path $P_{n}$ on $n \geq 3$ vertices, $\chi_{l a}\left(P_{n}\right)=3$.

- Cycle $C_{n}$ on $n \geq 3$ vertices, $\chi_{l a}\left(C_{n}\right)=3$.

- Friendship graph $F_{n}$ for $n \geq 2, \chi_{l a}\left(F_{n}\right)=3$.

- Friendship graph $F_{n}$ for $n \geq 2$ by removing an edge $e, \chi_{l a}\left(F_{n}-\{e\}\right)=3$.

- Complete bipartite graph $K_{m, n}$ for $m, n \geq 2, \chi_{l a}\left(K_{m, n}\right)=2$ if and only if $m \equiv n(\bmod 2)$.

- Complete bipartite graph $K_{2, n}$ for $n \geq 2, \chi_{l a}\left(K_{2, n}\right)=2$ for even $n \geq 2$ and $\chi_{l a}\left(K_{2, n}\right)=3$ for odd $n \geq 3$ or $n=2$.

- Graph $L_{n}$ for $n \geq 2$ that is obtained by inserting a vertex to each edge $v v_{i}, 1 \leq i \leq n-1$, of the star, $\chi_{l a}\left(L_{n}\right)=n+1$.

- Wheel $W_{n}$ of order $n+1$ for $n \geq 3, \chi_{l a}\left(W_{n}\right)=4$ if $n \equiv 1,3(\bmod 4), \chi_{l a}\left(W_{n}\right)=3$ if $n \equiv 2$ $(\bmod 4)$, and $3 \leq \chi_{l a}\left(W_{n}\right) \leq 5$ if $n \equiv 0(\bmod 4)$.

Furthermore, Arumugam et al. [1] showed that for any tree $T$ with $l$ leaves, $\chi_{l a}(T) \geq l+1$ and for the graph $H=G+\bar{K}_{2}$ where $G$ is a graph of order $n \geq 4$, then

$$
\chi_{l a}(G)+1 \leq \chi_{l a}(H) \leq \begin{cases}\chi_{l a}(G)+1 & \text { for } n \text { is even } \\ \chi_{l a}(G)+2 & \text { otherwise. }\end{cases}
$$

In this paper, we present the local antimagic chromatic number of unicyclic graphs such kite and cycle with two neighbour pendants. A graph is called unicyclic if it is connected and contains exactly one cycle. Therefore, a graph is unicyclic if and only if it is connected and has size equal to its order [4]. A kite, denoted by $K t_{n, m}$, consists of a cycle of length $n$ with a $m$-edge path (the tail) attached to one vertex [2].

\section{Main Results}

We start this section with a new result on the local antimagic chromatic number of the kite graph in the following theorem.

Theorem 2.1. For the kite $K t_{n, m}$ with $n \geq 3$ and $m \geq 1, \chi_{l a}\left(K t_{n, m}\right)=3$. 
Proof. Let $K t_{n, m}$ be the kite with $n \geq 3$ and $m \geq 1$. The vertex set of $K t_{n, m}$ is $V=\left\{u_{i} \mid 1 \leq\right.$ $i \leq n\} \cup\left\{v_{j} \mid 1 \leq j \leq m\right\}$ and the edge set is $E=\left\{u_{i} u_{i+1} \mid 1 \leq i \leq n-1\right\} \cup\left\{u_{n} u_{1}\right\} \cup\left\{u_{1} v_{1}\right\} \cup$ $\left\{v_{j} v_{j+1} \mid 1 \leq j \leq m-1\right\}$.

Label the edges of $K t_{n, m}$ using a bijection $f: E \rightarrow\{1,2, \ldots, n+m\}$ below.

$$
\begin{aligned}
& f\left(u_{i} u_{i+1}\right)= \begin{cases}\frac{m+1}{2}+\frac{i+1}{2}-1 & \text { for odd } m \text { and odd } i \\
\frac{m+i}{2} & \text { for even } m \text { and even } i \\
\frac{m+1}{2}+\left\lfloor\frac{n}{2}\right\rfloor+\left\lfloor\frac{n-1}{2}\right\rfloor-\frac{i}{2}+1 & \text { for odd } m \text { and even } i \\
\frac{m}{2}+\left\lfloor\frac{n}{2}\right\rfloor+\left\lfloor\frac{n+1}{2}\right\rfloor-\frac{i+1}{2}+1 & \text { for even } m \text { and odd } i\end{cases} \\
& f\left(u_{n} u_{1}\right)= \begin{cases}\left\lfloor\frac{n}{2}\right\rfloor+\frac{m+1}{2} & \text { for odd } m \\
\left\lfloor\frac{n+1}{2}\right\rfloor+\frac{m}{2} & \text { for even } m\end{cases} \\
& f\left(u_{1} v_{1}\right)= \begin{cases}\frac{m}{2} & \text { for even } m \\
\frac{m+1}{2}+n & \text { for odd } m\end{cases} \\
& f\left(v_{j} v_{j+1}\right)= \begin{cases}\frac{m-1}{2}-\frac{j+1}{2}+1 & \text { for odd } m \text { and odd } j \\
\frac{m-j}{2} & \text { for even } m \text { and even } j \\
\frac{m-1}{2}+\frac{j}{2}+n+1 & \text { for odd } m \text { and even } j \\
\frac{m}{2}+\frac{j+1}{2}+n & \text { for even } m \text { and odd } j\end{cases}
\end{aligned}
$$

It is easy to see that $f$ is a local antimagic labeling and the weight of vertices are

$$
\begin{aligned}
& w\left(u_{i}\right)= \begin{cases}\frac{3 m}{2}+2\left\lfloor\frac{n+1}{2}\right\rfloor+\left\lfloor\frac{n}{2}\right\rfloor & \text { for even } m \text { and } i=1 \\
n+m+1 & \text { for even }(m+i) \\
n+m & \text { for odd }(m+i) \\
\frac{3 m+3}{2}+\left\lfloor\frac{n}{2}\right\rfloor+n & \text { for odd } m \text { and } i=1\end{cases} \\
& w\left(v_{i}\right)= \begin{cases}n+m & \text { for even }(m+j) \\
n+m+1 & \text { for odd }(m+j)\end{cases}
\end{aligned}
$$

Thus, $\chi_{l a}\left(K t_{n, m}\right) \leq 3$. To show the lower bound, we can use the local antimagic chromatic number of cycle $C_{n}$ due to Arumugam et al. [1]. Since for $n \geq 3, \chi_{l a}\left(C_{n}\right)=3$ and the kite $K t_{n, m}$ contains a cycle $C_{n}$, it easy to see that $\chi_{l a}\left(K t_{n, m}\right) \geq 3$. Therefore $\chi_{l a}\left(K t_{n, m}\right)=3$.

Figure 1 shows an example of the local antimagic vertex coloring of the kite $K t_{5,6}$ with the local antimagic chromatic number equals to 3 .

We note that a $n$-pan graph, denoted by $P g_{n}$, is the graph obtained by joining a cycle graph $C_{n}$ to a singleton graph $K_{1}$ with a bridge. In other words, the $n$-pan graph is a special case of the kite graph $K t_{n, m}$ when $m=1$. Consequently,

Corollary 2.1. For the n-pan graph $P g_{n}$ with $n \geq 3, \chi_{l a}\left(P g_{n}\right)=3$.

In the next theorem, we present the local antimagic chromatic number of another unicyclic graph, that is the cycle with two neighbour pendants, as follows.

Theorem 2.2. For the cycle with two neighbour pendants $C p_{n}$ with $n \geq 3, \chi_{l a}\left(C p_{n}\right)=4$. 


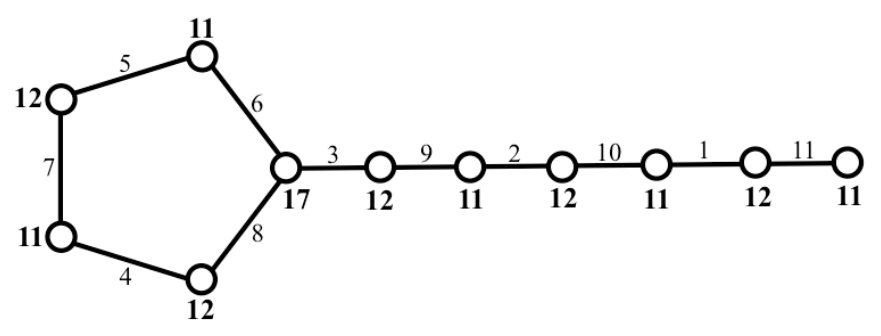

Figure 1. The local antimagic vertex coloring of $K t_{5,6}$ with $\chi_{l a}\left(K t_{5,6}\right)=3$

Proof. Let $C p_{n}$ be the cycle with two neighbour pendants $C p_{n}$ with $n \geq 3$. The vertex set of $C p_{n}$ is $V=\left\{u_{i} \mid 1 \leq i \leq n\right\} \cup\left\{v_{1}, v_{2}\right\}$ and the edge set is $E=\left\{u_{i} u_{i+1} \mid 1 \leq i \leq\right.$ $n-1\} \cup\left\{u_{n} u_{1}\right\} \cup\left\{u_{1} v_{1}, u_{2} v_{2}\right\}$.

Label the edges of $C p_{n}$ using a bijection $f: E \rightarrow\{1,2, \ldots, n+2\}$ below.

$$
\begin{array}{rlrl}
f\left(u_{i} u_{i+1}\right) & = \begin{cases}\frac{i+1}{2} & \text { for odd } i \\
n+1-\frac{i}{2} & \text { for even } i\end{cases} \\
f\left(u_{n} u_{1}\right) & =\left\lceil\frac{n+1}{2}\right\rceil & \\
f\left(u_{i} v_{i}\right)=n+i & \text { for } i=1,2
\end{array}
$$

It is easy to see that $f$ is a local antimagic labeling and the weight of vertices are

$$
\begin{aligned}
& w\left(u_{i}\right)= \begin{cases}\left\lfloor\frac{3 n+6}{2}\right. & \text { for } i=1 \\
2 n+3 & \text { for } i=2 \\
n+2 & \text { for odd } i \geq 3 \\
n+1 & \text { for even } i \geq 4\end{cases} \\
& w\left(v_{i}\right)=n+i \quad \text { for } i=1,2
\end{aligned}
$$

Thus $\chi_{l a}\left(C p_{n}\right) \leq 4$. To show the lower bound, we suppose that $f\left(u_{1} v_{1}\right)=m_{1}$ and $f\left(u_{2} v_{2}\right)=m_{2}$. Then $w\left(v_{1}\right)=m_{1}, w\left(v_{2}\right)=m_{2}, w\left(u_{1}\right)>m_{1}$ and $w\left(u_{2}\right)>m_{2}$. Clearly, $w\left(v_{1}\right) \neq w\left(v_{2}\right)$. Since $u_{1}$ is neighbour of $u_{2}$, then $w\left(u_{1}\right) \neq w\left(u_{2}\right)$. This implies that $\chi_{l a}\left(C p_{n}\right) \geq 4$. We conclude that $\chi_{l a}\left(C p_{n}\right)=4$.

Figure 2 shows an example of the local antimagic vertex coloring of the cycle with two neighbour pendants $C p_{6}$ with the local antimagic chromatic number equals to 4 .

\section{Conclusion}

Another family of unicyclic graph is a sun. A sun, denoted by $S u_{n}$, is a cycle on $n$ vertices $C_{n}$ with an edge terminating in a vertex of degree 1 attached to each vertex [2]. The local antimagic chromatic number of the sun $S u_{n}$ has not been discovered. Consequently, we have the following open problems.

Problem 1. Determine the local antimagic chromatic number of sun. 


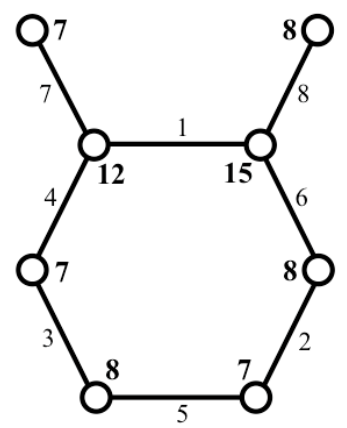

Figure 2. The local antimagic vertex coloring of $C p_{6}$ with $\chi_{l a}\left(C p_{6}\right)=4$

In general,

Problem 2. Determine the local antimagic chromatic number of some families of graph.

\section{Acknowledgement}

This research is supported by DRPM Ditjen Penguatan Risbang Kemenristekdikti under Penelitian Tim Pascasarjana 2017 research grant.

\section{Bibliography}

[1] S. Arumugam, K. Premalatha, M. Bača, A. Semaničová-Feňovčiková, Local antimagic vertex coloring of a graph, Graph and Combinatorics 33 (2017) 275-285.

[2] W. D. Wallis, E. T. Baskoro, Mirka Miller and Slamin, Edge-magic total labeling, Australasian Journal of Combinatorics 22 (2000) 177-190.

[3] L. Cai and J.A. Ellis, Edge colouring line graphs of unicyclic graphs, Discrete Applied Mathematics 36 (1992) 75-82.

[4] A.S. Pedersen and P.D. Vestergaard, The number of independent sets in unicyclic graphs, Discrete Applied Mathematics 152 (2005) 246-256. 